\title{
Quality of life in systemic lupus erythematosus and its measurement
}

\section{Agata Saletra, Marzena Olesińska}

Department of Systemic Connective Tissue Diseases, National Institute of Geriatrics, Rheumatology and Rehabilitation, Warsaw, Poland

\begin{abstract}
Systemic lupus erythematosus (SLE) is multi-system autoimmune rheumatic disorder with very broad clinical picture. Due to its generalized nature it influences all aspects of patient's life: physical, psychological and social well-being. With the development in diagnosis and treatment of SLE, median survival increased significantly over the past years. This article focused on the elements of quality of life, which are especially important for SLE patients, like body image, fatigue, family relations, disease impact on professional and social life. The quality of life could be measured with two different instruments: generic and disease-specific questionnaires. Generic ones are used to assess the quality of life of patients comparing to general population whereas specific questionnaires are designed to measure outcomes in one specific disease. The aim of the article is to describe HRQoL in SLE patients and the variables important for patients which have impact on it.
\end{abstract}

Key words: quality of life, systemic lupus erythematosus, outcome measures.

\section{Introduction}

Systemic lupus erythematosus (SLE) is a chronic, autoimmune inflammatory disease, with very broad and varied clinical picture. The disease can be oligosymptomatic, without significant influence on everyday life of a patient or can lead to recurrent aggravation and spread onto the vital organs, leading to disability or life threat. Thanks to the development in diagnosis and treatment of systemic lupus erythematosus, median survival increased significantly over the past years. As many as $95 \%$ of patients reach 5-year survival, whereas in the 1950 s this number amounted to $50 \%$ only [1]. Approximately $85 \%$ of early-diagnosed patients survive 10 years and $75 \%-20$ years [2]. Many studies show that the quality of life in patients with SLE is lower than in general population, independently of variables: measurement instruments (SF36, SF20, SF20+, and QOLS), ethnic origin or the size of the study group [3]. It refers both, to the evaluation of physical and mental health [2]. The quality of life in patients with SLE is comparable with the quali- ty of life in other chronic diseases: rheumatoid arthritis, Sjögren's syndrome or acquired immune deficiency syndrome (AIDS) [3].

The assessment of the quality of life should be inherent part of every medical appointment. It enables to holistic approach and proper communications with patients.

\section{Perception of the quality of life in systemic lupus erythematosus}

Systemic lupus erythematosus is a rare disease, difficult to diagnose. Therefore it appears that its prevalence is underestimated. The most probable data show that the prevalence of the disease among Caucasian Americans rates of 1.4 per 100,000 people [4]. The prevalence varies for different populations - African Americans and Asians suffer from SLE more often. The prevalence of disease in people of Afro-Caribbean ethnicity is reported to be 31,9 per 100,000 people [5]. About $80-90 \%$ of all systemic lupus erythematosus cases concern wom- 
en in the age of 20 to 40 years (mean age at diagnosis: 29 years) [6]. The disease causes many different clinical symptoms. Due to the generalized and chronic nature of the disease it influences in long term way all aspects of patient's quality of life. The quality of life is the patient's subjective perception of living with the disease. It specifies the approach towards the disease: ability to cope with everyday tasks and the level of satisfaction from the new life situation [7]. The following issues are particularly important: fatigue, inability to plan events/ meetings due to unforeseeable course of the disease, lack of disease understanding in the working environment. Numerous studies show that the disease activity does not correlate with patients' quality of life $[3,8]$. The issues relating to the impact of systemic lupus erythematosus on the quality of life that are of importance for the patients are presented in Figure 1.

\section{Diagnosis/course of the disease/prognosis}

Patients react differently to the diagnosis of systemic lupus erythematosus. Non-specific general changes (fatigue, weight loss, subfebrile states) as well as clinical symptoms that have developed within months, or even years, may hamper a diagnosis. A relief is a common reaction of patients to the diagnosis.

Long diagnosis, oligosymptomatic course of the disease, unspecific symptoms (weakness, fatigue), doubts of close persons and even physicians regarding the authenticity of declared symptoms cause frustration and concern in patients. Diagnosis and possibility to start the treatment calm them down. Others react to the diagnosis with anxiety and panic. Unforeseeable course of the disease can cause fear and feeling of insecurity regarding the future life. Some patients react with denying. They underestimate the diagnosis; it seems that it does not get through to them. Despite the symptoms,

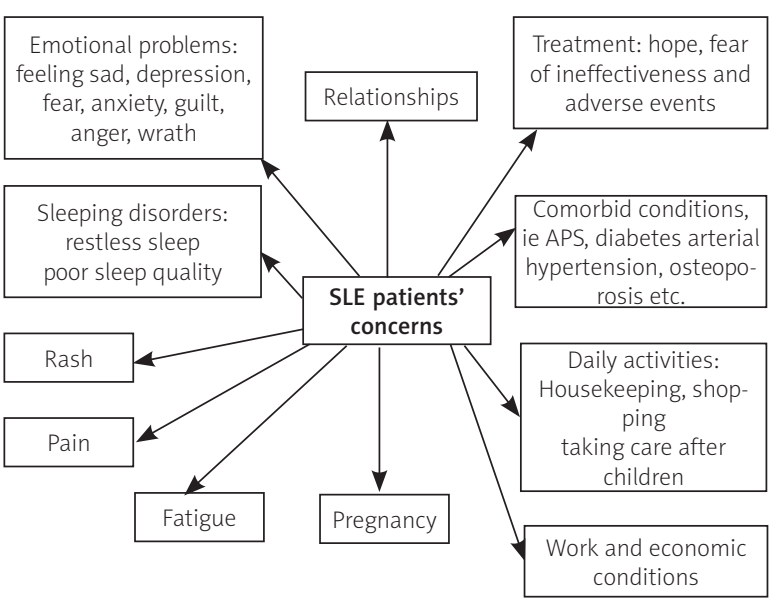

Fig. 1. SLE patients' concerns. they reluctantly agree to the treatment ("it is nothing serious", "it will certainly pass soon") [9]. Quality of life changes in the first 2 years of disease and is stable for the next 3 years [10].

Patients fear the course of the disease, another aggravation or spreading to new organs. It is often related to unpleasant experience at the beginning of the disease or serious aggravation from the past. Patients are worried about the recurrence of pain, intensification of weakness and fatigue. They fear the disease progression and even death [11]. This is usually explained by the lack of current information on SLE. Patients often obtain older data on the disease, in which the prognosis is very serious and the risk of mortality is high.

The acceptance of the disease usually demands time. Patients search for the information on SLE, observe their bodies and over time learn to recognize the symptoms of aggravation. It makes it easier to organize their work schedule and rearrange business issues for the time of indisposition. At some point patients find the strategy how to cope with disease. Most of them regards that keeping an active life and not thinking too much about consequences is the best way to fight with the disease [12]. Although even patient with great acceptance of disease have tough moments. Patients often react with angry and exasperation to situation, which in normal life would not cause such feelings [13].

Nearly $40 \%$ of patients cannot differentiate between the aggravation and overlapping symptoms of other disease. It could seem that with the duration of the disease and with age most patients would accept the impact of the disease on their lives. However most studies show negative correlation between $\mathrm{HRQOL}$ and the age of patients as well as contradictory results relating to the influence of disease duration on the quality of life [3, 9, 14].

\section{Body image}

In systemic lupus erythematosus the skin is frequently affected. Classic erythema on the face, discoid rash, lesions with tendency to scarring, skin atrophy and loss of hair are often unsightly and lower the self-esteem of a patient. These symptoms cause that people with SLE often feel embarrassed by their appearance. Patients also report bruising susceptibility and increased photosensitivity. They also fear weight gain due to the use of glucocorticosteroids [9, 15].

\section{Emotional disorders}

More than 2/3 of patients with SLE suffer from emotional disorders. The following were observed in the studies: feeling sad, depression, fear, anxiety, guilt, anger, wrath [15]. Many patients also experience the 
feeling of frustration, and a lack of confidence, independence control over ones life.

Most frequent symptoms include feeling of anxiety reported by over $70 \%$ of patients, mood disorders in $61.3 \%$ as well as depression in $50 \%$ of patients [16]. Anxiety is characterised by internal fear, lack of comfort and security. The feeling of anxiety appears already at the outset of the disease and can accompany patients with SLE throughout life. Most of concerns result from unpredictable course of the disease and the prognosis. The intensification of emotional disorders is particularly present at the time of disease aggravation. The disease triggers the feeling of helplessness and exhaustion by everyday struggle that the patient cannot win. Sometimes the symptoms of depression take form of somatic disorders: whole body pain, weight gain/loss, lack of interest in sex, amenorrhea or concentration and attention disorders $[8,9,14,16,17]$.

\section{Fatigue}

The fatigue and pain were the two most commonly reported symptoms affecting the quality of life of patients [18]. Constant fatigue has a devastating influence on many aspects of patients' life and undoubtedly constitutes a factor that worsens the quality of life. From 50 to $90 \%$ of patients with SLE experience constant fatigue and consider it the most common manifestation of the disease. Moskalewicz in her article thoroughly described the problems of young women struggling with fatigue and the need to perform the activities of everyday life [19].

The duration of the disease does not correlate with the intensification of fatigue [3]. Patients describe fatigue as unforeseeable, dominating, and controlling. The aetiology of fatigue in systemic lupus erythematosus is complex and has not yet been unambiguously defined. It is believed that the model of fatigue is similar to the one that occurs in rheumatoid arthritis described by Hawlett and is characterized by the disease, psychosocial and personal factors [20]. Most frequently described factors that influence the feeling of fatigue include: disease activity, sleep disorders, depression, anxiety, despondency, pain, emotional disorders, obesity, reduced physical activity, occurrence of coexistent diseases, vitamin D deficiency and the treatment used in SLE [21]. The studies on the impact of disease activity and coexistent diseases on the intensification of fatigue are ambiguous. There is no straight dependency between disease activity and intensification of fatigue. In one of the studies McKinley suggested an indirect relation between disease activity and fatigue. He claims that the disease activity correlates with increased prevalence of sleep disorders and depression, which leads to the increased prevalence of fatigue [22]. However patients with low disease activity also report constant fatigue. Most studies do not show that fatigue in SLE results only from the coexisting diseases (anaemia, hypothyroidism) [23].

Patients describe fatigue as paralyzing, insurmountable with sleep or rest. It limits everyday activities, often forces a patient to resign from earlier interests of hobbies (e.g. sports) or work. Patients feel helpless, powerless, sometimes angry and guilty. Fatigue is a symptom that cannot be seen. Patients are often frustrated that their relatives do not believe in such symptom. This generates conflicts relating to the division of household responsibilities, childcare or participation in social events. Physicians also have difficulties in understanding how severely fatigue impacts on patient's life. Over $80 \%$ of patients did not receive relevant support and advice from health care representatives. Despite the progress in treatment and new therapies available there is no medicine that could eliminate fatigue. Positive impact of non-pharmacological therapies, such as physical exercise or behavioural therapy that help overcome fatigue, was demonstrated [3, 9, 14, 17, 22].

\section{Sleep disorders}

Nearly $95 \%$ of patients with SLE report sleep disorders, mainly waking up frequently and restless sleep. They may result from the symptoms of basic disease: pain, stress, depression and side effects of medications (prednisone) as well as immunological changes caused by the disease [22].

\section{Pain}

Over $90 \%$ of patients suffer from joint pan - it is one of more frequent reasons why patients visit a physician. Pain significantly influences everyday functioning of patients, limits their abilities to discharge professional duties as well as household responsibilities, perform physical exercise and even personal activities (intimate hygiene) [15].

The implementation of adequate procedure that will mitigate pain is indispensable. Besides pain medication the following non-pharmacological therapies can be helpful: hot baths ensuring muscle relaxation, acupuncture and behavioural therapy. Additional orthopaedic equipment such as crutches, walking sticks, supports and walkers make moving around easier $[9,14]$.

\section{Physical activity/fitness}

The studies show that adequate level of physical activity has positive impact on physical, mental and social health in patients with chronic diseases, which increases their quality of life. Low level of physical activity in patients with SLE influences the development of: car- 
diovascular disease, osteoporosis, obesity, fatigue and sleep disorders. The implementation of regular training and exercise can decrease their occurrence. The study carried out by Robb-Nicholson showed that women with SLE had significantly lower oxygen consumption (45\% vs. $62 \%$ in the group of healthy women of the same age) [24]. It suggests that even patients with mild lupus could have insufficient aerobic capacity to perform professional, recreational (e.g. physical exercise) or everyday tasks. Patients with SLE limit their physical activity for many reasons. There is a fear of joint pain, bleeding (resulting from haematological disorders, increased risk of fracture. Photosensitivity makes it impossible for the patients to participate in sport activities in the open air. The obesity paradoxically discourages them to practise sport. The patients give up exercises due to depression and lack of interest. Depression has negative influence on patient's fitness and favours the development of further psychological issues. It was proved that swimming, riding a bike and walking decrease the level of depression in patients with SLE, which improves their wellbeing. All these factors inevitably lead to increasing disability of patients. Overcoming pain and stiffness of joints seems to be the most difficult. Over time, when the effects of exercise become visible, the patients rarely give up physical activity [25].

The introduction of training programmes, under the supervision of a physician and a physiatrist, individually adapted to patient's needs makes it possible to maintain proper physical fitness and performance as well as prevent injuries and other adverse reactions [9, 26].

\section{Work and economic conditions}

The concern for future career is fundamental. High disease activity, accompanying fatigue and pain prevent the patients from continuing work. Patients have often problem with being concentrated on the work and memory loss [18]. Absence and reduced work efficiency cause problems in keeping work or finding a new one. The patients are afraid to present yet another sick leave. Explaining the absence to the supervisor or colleagues who assume their responsibilities is often frustrating. It was showed that after 5 years of the disease 15-40\% of patients lose their jobs and after 10 and 15 years this percentage increases up to $36 \%$ and $52 \%$ respectively [4]. It involves the loss of income and deterioration of financial situation. The patients experience anger and frustration as they are not able to ensure a fair standard of living for their family. Due to unpredictable course of SLE manifestations many young patients fear that the disease will adversely affect their planned development path and additional responsibilities as well as make their starting career impossible to pursue [15]
The patients are also concerned about the costs of illness: medical costs and additional healthcare insurance costs [18].

Part-time work seems to be a solution. It makes it possible to remain in profession and carry out career path despite the limitations resulting from the disease. However, frequent sick leaves due to the aggravation of symptoms or hospitalization may present a problem. Moreover, additional costs such as medications, doctor's appointments or physical therapy arise. One of the possibilities is to use public health care or generic drugs [9].

Despite mainly negative impact of the disease on work some patients considered this situation beneficial - as a pretext for resigning from work that they did not enjoy or an opportunity to spend more time with children $[9,14]$.

\section{Relations with family and close persons}

The disease has negative impact also on family and social life. The patients do not fulfil their household and family responsibilities due to constant fatigue and bad mood. Women often report concern that they do not have the necessary strength to take care of children, neglect their duties as a mother, a wife and a hostess. Relatives often try to relieve them of their duties, which sometimes annoys and frustrates the patient who feels powerless and helpless. Despite the manifestations of the disease some patients decide to carry out their duties because they want to remain independent $[9,15]$. Patients point as the most problematic: moving heavy objects, doing laundry, shopping and taking care after children [18]. Relevant support ensured by the family makes it possible for the patient to avoid excessive burden. It was observed that the patients who are married or have life partner have higher HRQOL than single persons. However, it is not clarified whether it is thanks to more support from a partner or the fact that persons with low HRQOL have less chance to build a relationship [27].

Due to the increasing disability the patients fear that they will become a burden for the family. Fatigue and depression make them lose their interests. The disease have also negative impact on the partnership as the patients spend less quality time with their partners, who, in turn, have additional responsibilities. Patient fear rejection and separation. Young patients are afraid that they will not find a partner who will accept their disease and therefore they will not start a family [15].

Unforeseeable course of SLE is the reason why patients' social life is limited. "Invisibility" of symptoms, such as fatigue, can result in lack of understanding by friends as the patients cancel scheduled meetings. Due to photosensitivity the patients are often forced to re- 
sign from spending time with their families (activities in the open air, going to the beach). It frustrates them and increases their isolation $[9,14,15,17]$.

\section{Sexuality/intimate relations}

Few patients report sexual problems because for many persons it is an embarrassing issue. However, for most adults it is an issue that lowers their quality of life. Sexual dysfunctions are the result of both, physical and psychological problems.

Many symptoms of lupus that have negative impact on sexual activity can be observed. Joint pain is one of them. Patients often reduce their sex life because the pain occurs even with gentle movement. Muscle relaxation (sauna, hot bath) could be one of the solutions as well as selection of suitable, more passive position or time during the day when lupus manifestations are least annoying. The occurrence of Raynaud's symptom can also influence the manifestation of pain during the intercourse. The increase of blood circulation in the genital area reduces blood circulation in toes and fingers which exacerbates Raynaud's symptoms. Previously taken hot bath or increased temperature in the bedroom can significantly reduce this manifestation. Vaginal dryness, resulting from the coexisting Sjögren's syndrome, as well as ulceration of the mouth and vagina can be reduced with suitable medication and lubricants. Some antidepressants or high blood pressure medications can also cause sexual dysfunctions [9].

\section{Psychological and emotional problems}

Many patients with SLE struggle with low self-esteem resulting from the changes in physical appearance. Unsightly skin lesions and abdominal obesity (like in Cushing's syndrome) make the patients feel less attractive and concerned about negative reaction of their partner. Depression, constant fear and anxiety are well-known factors that cause the decline in sexual drive. The fear of passing the disease on to the offspring also makes the patients afraid of undertaking sexual activity [9].

\section{Pregnancy}

Patients with SLE fear that their children might suffer from the disease as well, therefore some give up family enlargement and parenthood completely [15]. Nearly half of the patients suffer from lupus aggravation during the pregnancy, therefore some women decide to have an abortion [9]. Pregnancy complications and anomalies in the foetus occur more often in the course of SLE than in general population. It is therefore of vital importance to prepare the patient for pregnancy and to ensure continuous monitoring during its course [6].

\section{Coexisting diseases}

LULA-Study, carried out in German patients with SLE, presents most frequent coexisting diseases. The following were identified: hypertension (30\% of patients, $41 \%$ after 4 years), arthritis (27.5\%), osteoporosis (27.9\%), psychiatric disorders (25\%), skin lesions (21\%), nephropathy (20\%), thrombosis (20\%) and hyperlipidemia (17.6\%). The number of patients with hyperlipidemia increased twice after four years of observation (32\%) [27]. Increased mortality occurs more often in patients with SLE in comparison with general population, and is mainly due to cardiovascular diseases. Hyperlipidemia and hypertension are independent factors of the increased risk of cardiovascular events. The study showed that both lupus nephritis and psychiatric disorders did not influence patients' quality of life, however psychiatric disorders can correlate with the scores of mental component summary (MCS) [27].

\section{Treatment}

The implementation of treatment often evokes mixed feelings. The hope of avoidance the manifestations of SLE, daily routine of taking drugs and trust with rheumatologist's recommendations is the main reason for adherence to therapy [12].

On the one hand the patients are optimistic that the symptoms of the disease will be controlled and its activity reduced, on the other hand they fear side effects of the therapy. They are most afraid of glucocorticoids and their long-term side effects, such as osteoporosis, weight gain, cataract and glaucoma [15]. There is a continuous hope for new therapies [9]. Patients are most often treated with glucocorticoids as monotherapy or combined with hydroxychloroquine, NSAIDs and immunosuppressants [27]. The studies show that immunosuppressants (methotrexate, cyclophosphamide) reduce disease activity, but they do not influence the quality of life improvement. Similarly, hydroxychloroquine limits the aggravations and increases survival rate, but it does not correlate with the increased quality of life. One of the studies proved that after a year of using belimumab positive relation with quality of life and fatigue reduction was observed $[27,28]$. Nearly half of the patients $49.8 \%$ out of 707 patients who participated in the study $[28,29]$ decided to use alternative medicine. Alternative therapy was used mainly by young, educated persons from developing countries. Despite negative impact on the quality of life and harmful effects on health $40 \%$ of patients with SLE from Western countries use complementary alternative therapies [28].

The use of non-pharmacological therapies has positive influence on the quality of life. It was indicated that 
the implementation of cognitive behavioural therapies improved patient's functioning, limited physical and psychological manifestations of the disease as well as emotional disorders and had positive impact on abilities/ social life. It improved patients' self-esteem, reduced the impact of the disease on life and improved the general condition as well as decreased the pain $[8,14]$.

Relevant support provided by the physician and healthcare professionals is of great importance for the patient. It was showed that it correlates with the increased quality of life [11]. Insufficient knowledge on SLE among primary care physicians, difficulties in seeing a specialist, inadequate accessibility of psychologists specializing in providing assistance to patients with chronic diseases have negative impact on patients' quality of life $[6,15]$.

Although patients are seemed to be satisfied with relationship with their rheumatologists. However many of them do not share their problems and concerns on their visit. It may happen because they simply forgot about it or there is no chance to discuss. They emphasise that physician often give many information but rarely let the patients talk about their concerns.

The patients also point out that physician do not take too much care to problem with their appearance [12].

\section{Positive aspects}

Acceptance and adjustment to life with a disease is difficult, in particular for young persons, and requires time. Unpredictable and dominating impact of the disease gives new priority to patients' life. Despite the limitations and inconveniences of the disease, patients also indicate positive aspects. They gained knowledge on their body and the disease, learned how to function with the disease and overcome the obstacles of everyday life. They were more grateful and enjoyed the pleasures of simple moments $[14,17]$. The disease became also a pretext for resigning from work or activities that the patients did not enjoy as well as to meet new, valuable people [15]. Patients started to focus on important things that mattered in their life [15].

\section{Quality of life}

World Health Organization (WHO) defines the quality of life as “individuals' perception of their position in life in the context of the culture and value systems in which they live and in relation to their goals, expectations, standards and concerns" [30]. It comprises all aspects of human life - four basic: somatic state, mental wellbeing, social relationships and physical fitness as well as supplementary: the impact of environment, economic status, functioning in social roles, sexuality, and body image. From the point of view of medical science a more narrow term of "health-related quality of life" (HRQOL) is used, which is based on WHO definition and defines health as "a state of complete physical, mental, and social well-being, not merely the absence of disease" [31, 32]. HRQOL rate is based on patient's self-evaluation of the impact of the disease and treatment on physical, psychological and social functioning as well as patient's satisfaction relating to the functioning on all levels, control over the disease and side effects resulting from the treatment [33].

The assessment of SLE influence on patients' life includes three factors: assessment of disease activity, organ damage in the course of the disease and the quality of life $[34,35]$. Both, the disease activity and the level of organ damage are assessed by a physician. The assessment of the disease activity is carried out with the help of different scores (e.g. SELENA-SLE, BILAG index and the level of organ damage is assessed with the use of SLICC ACR Damage Index. Thanks to the quality of life assessment, a physician can evaluate a patient holistically, taking into account not only clinical condition, but also emotional and mental wellbeing as well as socio-economic factors. It is important in many aspects of patient care, for instance, in selecting therapeutic management. The implementation of aggressive treatment reduces the disease activity or the level of organ damage; however the side effects influence the patient's wellbeing and functioning in family life and at work, resulting in failure to follow doctor's recommendations [36]. Quality of life assessment constitutes a distinct value in the assessment of a chronic disease, facilitates the planning and organization of patient care and ensures proper relationship between a physician and a patient.

\section{Methods of quality of life assessment}

Three types of questionnaire are used to assess the quality of life: general (generic), specific and mixed. Generic questionnaires are used to assess the quality of life of healthy persons and persons with a disease in general population (SF-36, EQ-5D, WHOQOL). They do not take into account characteristic variables of a given disease, however they allow for comparison of the quality of life in various disease units. The lack of sensitivity towards characteristics of a given diseases makes general questionnaires imperfect. It is important to include the following elements in the quality of life assessment in patients with SLE: sleep disorders, fatigue, body image and social relations [15]. Specific questionnaires (e.g. SLEQOL, LupusQOL, and LupusPro) are developed by specialists in a given area and with patients' participation, therefore include many issues that are relevant for patients with SLE. 
Although the specific questionnaires have been designed based on important aspects for patients, these are not totally perfect instruments. The questionnaires should be sensitive enough to detect change in HRQoL. There should be further studies to determine what is the minimal meaningful change in a score, which has influence on HRQoL.

Mixed questionnaires include elements of both questionnaires mentioned above and are used for specific diseases in clinical trials [32].

The choice of HRQol measure should be chosen depend on the research question and studied population. It may be useful to use both type of questionnaires. If it is a need to compare between different diseases or with normal controls, then generic scale would be more appropriate. In case of examination specific for SLE patients issues (e.g. response to therapy) the disease-specific questionnaires would be more suitable.

\section{Generic questionnaires}

The following questionnaires are most commonly used in patients with systemic lupus erythematosus: SF-36, SF-20, EQ-5D, WHOQOL and VAS.

SF-36 survey is an American instrument that allows for general quality of life assessment in the population of healthy persons and patients. It comprises 11 questions that include 36 items. The following aspects can be assessed: physical functioning, activity in everyday functioning as well as at work, social functioning, bodily pain, fatigue, exhaustion, depression or anxiety. SF-36v2 survey includes an additional question about changes in the state of health within the last 12 months. The method of assessment is diversified and depending on a question 3, 5 or 6-point Likert scale is used. The maximum of 100 points can be obtained. The lower the score the lower quality of life [37]. SF-36 survey is recognized as a relevant indicator to assess the quality of life in Caucasian patients with SLE [29].

SF-20 survey is a shorter version of SF-36 survey. It comprises 20 items divided into 6 domains relating to physical functioning (PF), role functioning (RF), social functioning (SF), mental health $(\mathrm{MH})$, health perception (HP) and bodily pain (BP). The results are assessed in 1-10 scale and then multiplied by 100 [38].

EQ-5D questionnaire includes 5 questions on mobility, self-care, usual activities, pain, and depression. EQ-5D is one of the most commonly used generic health status measurement, and its good validity and reliability have been reported in various health conditions. The questionnaire has the advantage of being quick to complete [39].

WHOQOL in its full version (WHQORL-100) comprises 100 questions, whereas shorter version (BREF), which is more often used, includes 26 questions. WHOQOL-BREF measures the following quality of life domains: physical health, psychological health, social relationships, and environment [40].

Visual Analogue Scale (VAS) is a visual instrument that uses $100 \mathrm{~mm}$ analogue scale on which a patient (and a physician) indicates, with a vertical line, a position that corresponds to the intensity of an assessed phenomenon. The result is measured from the startpoint of the scale to the indicated position. The score pf $0 \mathrm{~mm}$ means lack of disease and $100 \mathrm{~mm}$ means the highest intensity of an assessed phenomenon.

\section{Specific questionnaires}

LupuSQOL is a measure developed by McElhone et al. in 2007 to assess the quality of life of patients with SLE in Great Britain. It comprises 34 items grouped into 8 domains: physical health, pain, planning, intimate relationships, burden to others, emotional health, body image, fatigue. Each item is assessed by a patient with the use of 4-point Likert scale (0-4, where 0 means "all the time" and 4 - "never"). Each domain is assessed separately: mean raw domain score is divided by four and then multiplied by 100. The obtained score determines the patient's quality of life in a given domain (0 - worst HRQoL and 100 - best HRQOL). Spanish version of the questionnaire was validated as well as the version for American population. The LupusQoL has been also translated into 77 languages for use in 51 countries [41].

SLEQOL is a measure developed by Leong et al. that includes 40 items grouped into 6 domains: physical functioning, activities, symptoms, treatment, mood and self-image. The questions are answered with the use of 7-point Likert scale. The total score is the sum of all points (from 40 to 280). The higher the score, the worse quality of life [42].

SSC is an instrument developed as a result of cooperation between physicians and patients and includes 38 statements on clinical symptoms and treatment of systemic lupus erythematosus. The answers are dichotomous (Yes or No). The higher the score, the worse patient's quality of life. At present it is considered a list of symptoms and not the proper quality of life indicator as its sensitivity was validated in the group of patients that was too small [43].

LupusPro tool was developed based on the feedback and final assessment of patients with SLE in American population, of varied ethnic backgrounds and either gender. It comprises 44 items measuring health-related (HRQOL) and non-health-related domains (non-HRQOL). The first domain includes: lupus symptoms, physical health (physical function, role physical), pain/vitality, emotional health (emotional function and role emotional), body image, cognition, procreation and lupus medications. The non-health-related domains include available 
Table I. SLE patient's concerns

\begin{tabular}{|c|c|c|c|c|c|c|}
\hline Measure & $\begin{array}{l}\text { Number } \\
\text { of items }\end{array}$ & $\begin{array}{l}\text { Time to } \\
\text { complete }\end{array}$ & Content & Score interpretation & $\begin{array}{l}\text { Ability to } \\
\text { change }\end{array}$ & $\begin{array}{c}\text { Validation } \\
\text { and Polish } \\
\text { language } \\
\text { version }\end{array}$ \\
\hline \multicolumn{7}{|c|}{ SLE-specific questionnaires } \\
\hline LupusQoL & 34 & $<10 \min$ & $\begin{array}{l}\text { Physical health, pain, planning, intimate } \\
\text { relationships, burden to others, emotio- } \\
\text { nal health, body image, fatigue }\end{array}$ & $\begin{array}{l}\text { 0-100 } \\
\text { Higher score indicates } \\
\text { better QoL }\end{array}$ & $x$ & \\
\hline SLEQoL & 40 & $<5 \min$ & $\begin{array}{l}\text { Physical functioning, activities, symp- } \\
\text { toms, treatment, mood and self-image }\end{array}$ & $\begin{array}{l}\text { 40-280 } \\
\text { Higher score indicates } \\
\text { worse QoL }\end{array}$ & Partial & \\
\hline SSC & 38 & $2-3 \mathrm{~min}$ & $\begin{array}{l}\text { List of SLE and treatment-related symp- } \\
\text { toms and their burden on the patient }\end{array}$ & $\begin{array}{l}\text { Higher score indicates } \\
\text { worse QoL }\end{array}$ & $x$ & \\
\hline LupusPro & 43 & $<10 \min$ & $\begin{array}{l}\text { Lupus symptoms, physical health, pain/ } \\
\text { vitality, emotional health, body image, } \\
\text { cognition, procreation and lupus medi- } \\
\text { cations, available social support and cop- } \\
\text { ing, desires and goals and medical care } \\
\text { satisfaction. }\end{array}$ & $\begin{array}{l}\text { 0-100 } \\
\text { Higher score indicates } \\
\text { worse QoL }\end{array}$ & $x$ & $\begin{array}{l}\text { Process of } \\
\text { validation } \\
\text { in Poland }\end{array}$ \\
\hline L-Qol & 25 & $<5 \min$ & $\begin{array}{l}\text { List of items assessing the impact of SLE } \\
\text { and its treatment on the patient }\end{array}$ & $\begin{array}{c}\text { 0-25 } \\
\text { Higher score indicates } \\
\text { worse QoL }\end{array}$ & $x$ & \\
\hline \multicolumn{7}{|c|}{ Generic questionnaires } \\
\hline SF-36 & 36 & $<10 \min$ & $\begin{array}{l}\text { Physical functioning, activity in everyday } \\
\text { functioning as well as at work, social } \\
\text { functioning, bodily pain, fatigue, exhaus- } \\
\text { tion, depression or anxiety }\end{array}$ & $\begin{array}{l}\text { 0-100 } \\
\text { Higher score indicates } \\
\text { better QoL }\end{array}$ & partial & $\checkmark$ \\
\hline SF-20 & 20 & $5 \mathrm{~min}$ & $\begin{array}{l}\text { Physical functioning (PF), role function- } \\
\text { ing (RF), social functioning (SF), mental } \\
\text { health }(\mathrm{MH}) \text {, health perception }(\mathrm{HP}) \text { and } \\
\text { bodily pain (BP }\end{array}$ & $\begin{array}{l}\text { Higher score indicates } \\
\text { better QoL }\end{array}$ & $x$ & \\
\hline EQ-5D & $\begin{array}{l}5 \text { and } \\
\text { VAS } \\
\text { scale }\end{array}$ & $2-5 \min$ & $\begin{array}{l}\text { Mobility, self-care, usual activities, pain, } \\
\text { and depression }\end{array}$ & $\begin{array}{l}\text { Higher score indicates } \\
\text { better QoL }\end{array}$ & $\checkmark$ & $\checkmark$ \\
\hline $\begin{array}{l}\text { WHOQOL- } \\
\text { Bref }\end{array}$ & 26 & $<10 \min$ & $\begin{array}{l}\text { Physical health, psychological health, so- } \\
\text { cial relationships, and environment }\end{array}$ & $\begin{array}{l}\text { Higher score indicates } \\
\text { better QoL }\end{array}$ & $x$ & \\
\hline
\end{tabular}

social support and coping, desires and goals and medical care satisfaction. The questionnaire takes account of changes arising in time. It is considered a valid and reliable tool for the assessment of patients with SLE [44].

$\mathrm{L}-\mathrm{QOL}$, contrary to the above-mentioned, is a one-dimensional questionnaire that comprises 25 items assessing the impact of the disease (SLE) on patient's treatment. Each question is answered with Yes or No. The quality of life indicator is obtained by adding up all the points (from 0 to 25). Higher score indicates worse quality of life (Table I) [44].

\section{Conclusions}

Systemic lupus erythematosus, due to its systemic nature, can affect all aspects of patient's life. The quality of life in patients with SLE is lower than in general popula- tion and comparable with other chronic diseases. Generic and specific questionnaires, taking into account elements specific for patients with SLE: body image, fatigue, family relations, impact on professional and social life are used to assess the quality of life in patients with SLE. The quality of life assessment should constitute a necessary part of each medical appointment. It facilitates the planning of patient care, holistic approach as well as reduces the distance between a physician and a patient.

The authors declare no conflict of interest.

\section{References}

1. Zimmerman-Górska I. Postępy w reumatologii klinicznej. Wyd. Lek. PZWL 2014; 129-159. 
2. Rinaldi S, Doria A, Salaffi F, et al. Health-related quality of life in Italian patients with systemic lupus erythematosus. I. Relationship between physical and mental dimension and impact of age. Rheumatology (Oxford) 2004; 43: 1574-1579.

3. McElhone K, Abbott J, Teh LS. A review of health related quality of life in systemic lupus erythematosus. Lupus 2006; 15 : 633-643.

4. Danchenko N, Satia JA, Anthony MS. Epidemiology of systemic lupus erythematosus: a comparison of worldwide disease burden. Lupus 2006; 15: 308-318.

5. Lim S, Drenkard C. Chapter 2. The epidemiology of lupus. In: Wallace DJ, Hahn B. Dubois' Lupus Erythematosus and Related Syndromes. Saunders, Philadelphia 2013; 14.

6. Toczeń rumieniowaty układowy w Polsce. Raport. On-line. Majdan M (ed.). Warszawa 2012; Access: 4.02.2016.

7. Gotay CC, Korn EL, McCabe MS, et al. Quality-of-life assess ment in cancer treatment protocols: research issues in protocol development. J Natl Cancer Inst 1992; 84: 575-579.

8. Navarrete-Navarrete N, Peralta-Ramírez MI, Sabio JM, et al. Quality-of-life predictor factors in patients with SLE and their modification after cognitive behavioural therapy. Lupus 2010; 19: 1632-1639.

9. Phillips RH. Coping with Lupus. Avery Penguin Putnam, New York 2001

10. Urowitz M, Gladman DD, Ibañez D, et al. Changes in quality of life in the first 5 years of disease in a multicenter cohort of patients with systemic lupus erythematosus. Arthritis Care Res (Hoboken) 2014; 66: 1374-1379.

11. Sutcliffe N, Clarke A, Levinton C, et al. Associates of health status in patients with systemic lupus erythematosus. J Rheumatol 1999; 26: 2352-2356.

12. Farinha F, Freitas F, Águeda A, et al. Concerns of patients with systemic lupus erythematosus and adherence to therapy a qualitative study. Patient Prefer Adherence 2017; 11: 12131219.

13. Baranowska M. To jest wasze życie. Być sobą w chorobie przewlekłej. Wyd. Czarne, Białystok 2011.

14. Wallace D. The Lupus Book. A Guide for Patients and Their Familes. Oxford University Press, Oxford 2005

15. McElhone K, Abbott J, Gray J, et al. Patient perspective of systemic lupus erythematosus in relation to health-related quality of life concepts: a qualitative study. Lupus 2010; 19: 1640-1647.

16. Bauernfeind B, Aringer M, Prodinger B, et al. Identification of relevant concepts of functioning in daily life in people with systemic lupus erythematosus: A patient Delphi exercise. Arthritis Rheum 2009; 61: 21-28.

17. Mattsson M, Möller B, Stamm T, et al. Uncertainty and opportunities in patients with established systemic lupus erythematosus: a qualitative study. Musculoskeletal Care 2012; 10: 1-12.

18. Holloway L. Patient-reported outcome measures for systemic lupus erythematosus clinical trials: a review of content validity, face validity and psychometric performance. Health Qual Life Outcomes 2014; 12: 116.

19. Moskalewicz B. W poszukiwaniu kulturowych przyczyn zmęczenia u chorej na toczeń rumieniowaty układowy. Reumatologia 2007; 45: 18-26.
20. Hawlett S, Chalder T, Choy E, et al. Fatigue in rheumatoid arthritis. Time for conceptual model. Rheumatology (Oxford) 2011; 50: 1004-1006.

21. Ahn GE, Ramsey-Goldman R. Fatigue in systemic lupus erythematosus. Int J Clin Rheumatol 2012; 7: 217-227.

22. McKinley PS, Ouellette SC, Winkel GH. The contributions of disease activity, sleep patterns, and depression to fatigue in systemic lupus erythematosus. Arthritis Rheum 1995; 38: 826-834.

23. Cleanthous S, Tyagi M. What do we know about self-reported fatigue in systemic lupus erythematosus? Lupus 2012; 21 : 465-476.

24. Robb-Nicholson L, Daltroy L, Eaton H, et al. Effects of aerobic conditioning in lupus fatigue: a pilot study. $\mathrm{Br} J$ Rheumatol 1989; 28: 500-505.

25. Mancuso CA, Perna M, Sargent AB, et al. Perceptions and measurements of physical activity in patients with systemic lupus erythematosus. Lupus 2011; 20: 231-242.

26. Ayán C, Martín V. Systemic lupus erythematosus and exercise, Lupus 2007; 16: 5-9.

27. Tamayo T, Fischer-Betz R, Beer S, et al. Factors influencing the health related quality of life in patients with systemic lupus erythematosus: long-term results (2001-2005) of patients in the German Lupus Erythematosus Self-Help Organization (LULA Study). Lupus 2010; 19: 1606-1613.

28. Toloza SM, Sequeira W, Jolly M. Treatment of lupus: impact on quality of life. Curr Rheumatol Rep 2011; 13: 324-337.

29. Stoll T, Gordon C, Seifert B, et al. Consistency and validity of patient administered assessment of quality of life by the MOS SF-36; its association with disease activity and damage in patients with systemic lupus erythematosus. J Rheumatol 1997; 24: 1608-1614.

30. WHO: WHOQOL. Measuring Quality of Life. Division of mental health and prevention of substance abuse. WHO 1997.

31. WHO: Constitution of the World Health Organization, Basic Documents. supl. 2006.

32. Klocek M. Kwestionariusze jakości życia w chorobach układu sercowo-naczyniowego. In: Jakość życia w chorobach sercowo-naczyniowych. Kawecka-Jaszcz K, Klocek M, TobiaszAdamczyk B (eds.). Termedia, Poznań 2006.

33. Schipper H. Quality of Life: principles of the clinical paradigm. J Psychosocial Oncol 1990; 8: 171-185.

34. Griffiths B, Mosca M, Gordon C. Assessment of patients with systemic lupus erythematosus and the use of lupus disease activity indices. Best Pract Res Clin Rheumatol 2005; 19: 685708.

35. Gotay CC, Korn EL, McCabe MS, et al. Quality-of-life assessment in cancer treatment protocols: research issues in protocol development. J Natl Cancer Inst 1992; 84: 575-579.

36. Jaeschke R. Evidence based medicine (EBM), czyli praktyka medyczna oparta na wiarygodnych i aktualnych publikacjach (POWAP). Odcinek 8. Określanie i mierzenie jakości życia związanej ze zdrowiem. Medycyna Praktyczna 1999; 4: 155162.

37. Tylka J, Piotrowicz R. Quality of life SF-36 questionnaire - the Polish version. Kardiol Pol 2009; 67: 1166-1169. 
38. Stewart AL, Hays RD, Ware JE Jr. The MOS short-form general health survey. Reliability and validity in a patient population. Med Care 1988; 26: 724-735.

39. Rabin R, de Charro F. EQ-5D: A measure of health status from the EuroQol Group. Ann Med 2001; 33: 337-334.

40. Leong KP, Kong KO, Thong BY, et al. Development and preliminary validation of a systemic lupus erythematosus-specific quality-of-life instrument (SLEQOL). Rheumatology (Oxford) 2005; 44: 1267-1276.

41. The World Health Organization Quality of Life assessment (WHOQOL): position paper from the World Health Organization. Soc Sci Med 1995; 41: 1403-1409.

42. McElhone K, Abbott J, Shelmerdine J, et al. Development and validation of a disease-specific health-related quality of life measure, the Lupus Qol, for adults with systemic lupus erythematosus. Arthritis Rheum 2007; 57: 972-979.

43. Yee CS, McElhone K, Teh LS, et al. Assessment of disease activity and quality of life in systemic lupus erythematosus - new aspects. Best Pract Res Clin Rheumatol 2009; 23: 457-467.

44. Jolly M, Pickard AS. Disease-specific patient reported outcome tools for systemic lupus erythematosus. Semin Arthritis Rheum 2012; 42: 56-65. 